\title{
Closed Wedge Distal Femoral Osteotomy with a Polyaxial Locking Plate Designed for the Proximal Tibia: Minimum 5-Year Outcomes
}

Ryuichi Nakamura, $\mathrm{MD}^{1}$, Kenji Fujita, $\mathrm{MD}^{2}$, Rei Omi, MD¹, Kazunari Kuroda, MD ${ }^{1}$, Masaki Takahashi, MD ${ }^{1}$, Kazumi Ikebuchi, $\mathrm{MD}^{1}$, Hitoshi Nishimura, $\mathrm{MD}^{3}$, and Yasuo Katsuki, $\mathrm{MD}^{1}$

${ }^{1}$ Department of Orthopaedic Surgery, Yawata Medical Center, Komatsu; ${ }^{2}$ Department of Orthopaedic Surgery, Kanazawa University, Kanazawa; ${ }^{3}$ Department of Rehabilitation, Yawata Medical Center, Komatsu, Japan

Since distal femoral varus osteotomy (DFO) -specific plates had not been available in Japan before 2015, we performed DFO using a plate for tibia. The purpose of this study was to elucidate the efficacy and problems associated with the non-specific plate in DFO. We used NCB-PT plates (Zimmer Inc.) in the upside-down position and the minimum 5-year outcomes were evaluated. The mean preoperative weight bearing line ratio and Japanese Orthopaedic Association score improved from $97.6 \% \pm 35.8 \%$ and $68.0 \pm 11.5$, respectively, to $44.0 \% \pm 16.1 \%$ and $82.0 \pm 7.6$, respectively, 1 year postoperatively and to $42.8 \% \pm 15.7 \%$ and $86.0 \pm 8.2$, respectively, 5 years postoperatively. The flexion range decreased from $149.0^{\circ} \pm 6.5^{\circ}$ to $138.0^{\circ} \pm 5.7^{\circ}$ 1 year postoperatively and to $135.0^{\circ} \pm 20.9^{\circ} 5$ years postoperatively. Although DFO using the NCB-PT plate provided mid-term benefits, it resulted in a loss of knee flexion, possibly due to excessive coverage of the medial femoral epicondyle.

Keywords: Knee, Osteoarthritis, Osteotomy, Plate, Complication

Valgus deformity with lateral compartment knee osteoarthritis is much less common than varus deformity with medial compartment knee osteoarthritis. Thus, there are fewer studies reporting outcomes of distal femoral varus osteotomy (DFO) ${ }^{1,2)}$ than of high tibial osteotomy (HTO) for medial osteoarthritis. Unfortunately, before the TomoFix Medial Distal Femoral Plate (TomoFix MDF; DePuy Synthes GmbH, Solothurn, Switzerland) (Fig. 1) was launched in Japan, plates specifically designed for medial closed wedge distal femoral varus osteotomy (CWDFO) were not available. Therefore, from 2010 to 2014, we performed CWDFO

Received February 13, 2017; Revised (1st) April 8, 2017;

(2nd) May 13, 2017; Accepted May 13, 2017

Correspondence to: Ryuichi Nakamura, MD, PhD

Department of Orthopaedic Surgery, Yawata Medical Center, 12-7

Yawata, Komatsu, Ishikawa 923-8551, Japan

Tel: +81-761-47-1212, Fax: +81-761-47-1941

E-mail: ryu-nakamura@msj.biglobe.ne.jp

This is an Open Access article distributed under the terms of the Creative Commons Attribution Non-Commercial License (http://creativecommons.org/licenses/by-nc/4.0/) which permits unrestricted non-commercial use, distribution, and reproduction in any medium, provided the original work is properly cited.

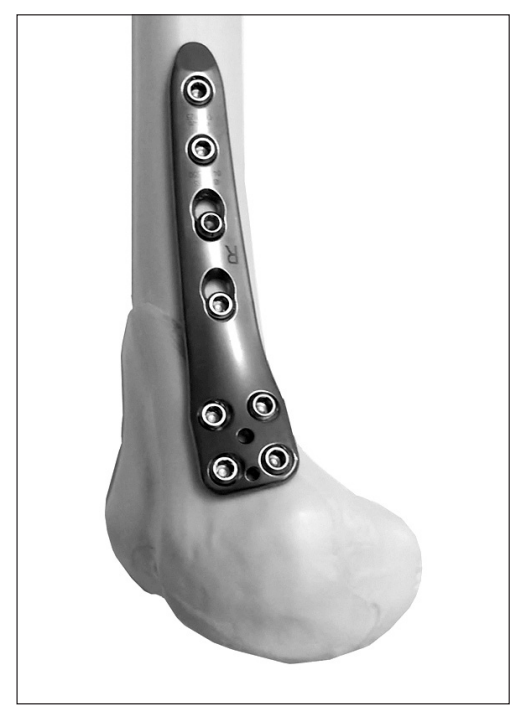

Fig. 1. Fitting of the TomoFix Medial Distal Femoral Plate (MDF; DePuy Synthes $\mathrm{GmbH}$ ) on the medial distal femur. The distal end of the plate is located above the medial femoral epicondyle. 
with the Non-Contact Bridging Plate for Proximal Tibia (NCBPT; Zimmer Inc., Winterthur, Switzerland), placing the plate upside down. Although not specifically designed for CWDFO, when used in this position, the NCB-PT provided good contact with the medial distal femur (Fig. 2), and its polyaxial locking system $^{3)}$ was easy to use and allowed for correct screw placement.

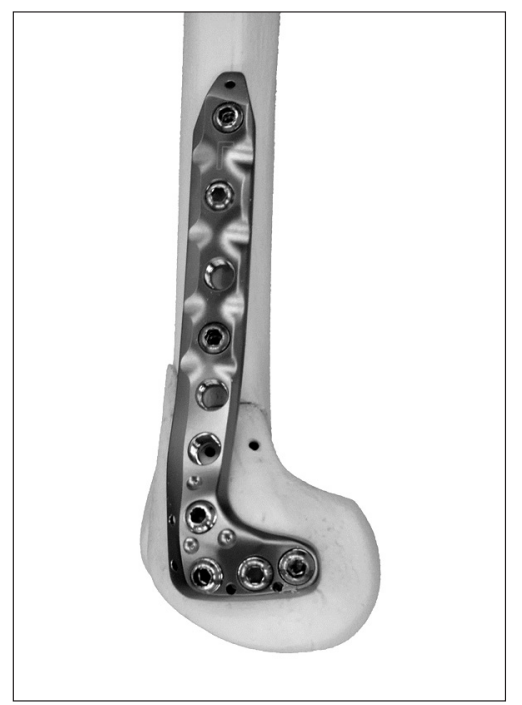

Fig. 2. Fitting of the Non-Contact Bridging Plate for Proximal Tibia (NCB-PT; Zimmer Inc.) in the upside-down position on the medial distal femur. The plate fits well on the modeled bone after medial closedwedge distal femoral osteotomy. It fully covers the medial femoral epicondyle.
The purpose of this study was to evaluate the mid-term results of a minimum 5-year follow-up of CWDFO using the NCB-PT and to elucidate the problems associated with the use of a plate not specifically designed for CWDFO. TomoFix MDF has been available in Japan since 2015, but we believe that some of the pitfalls of CWDFO may be more evident and more easily clarified by our description of the use of a plate not specifically designed for CWDFO.

\section{Case Report}

Five patients who were treated with CWDFO using the NCBPT between 2010 and 2012 and had a minimum of 5 years of follow-up were investigated. We aimed for a corrected weight bearing line (WBL) percentage $e^{4)}$ of $40 \%-50 \%$, except in case 2 . Patients began active and passive range of motion on the first postoperative day and the standard protocols for partial and full weight bearing were started 4 and 8 weeks after surgery, respectively. All plates were removed one year after surgery.

A full-length anteroposterior radiograph of the lower extremity with full weight bearing was obtained preoperatively and 1 and 5 years postoperatively. The Japanese Orthopaedic Association (JOA) score ${ }^{5}$ and range of knee flexion were assessed preoperatively and 1 and 5 years after surgery. Both the radiographic and clinical outcomes at 1 year after surgery were evaluated before plate removal. The data for each case are presented in Table 1 .

The preoperative mechanical proximal tibial angle (mMPTA)

Table 1. Details of Each Case

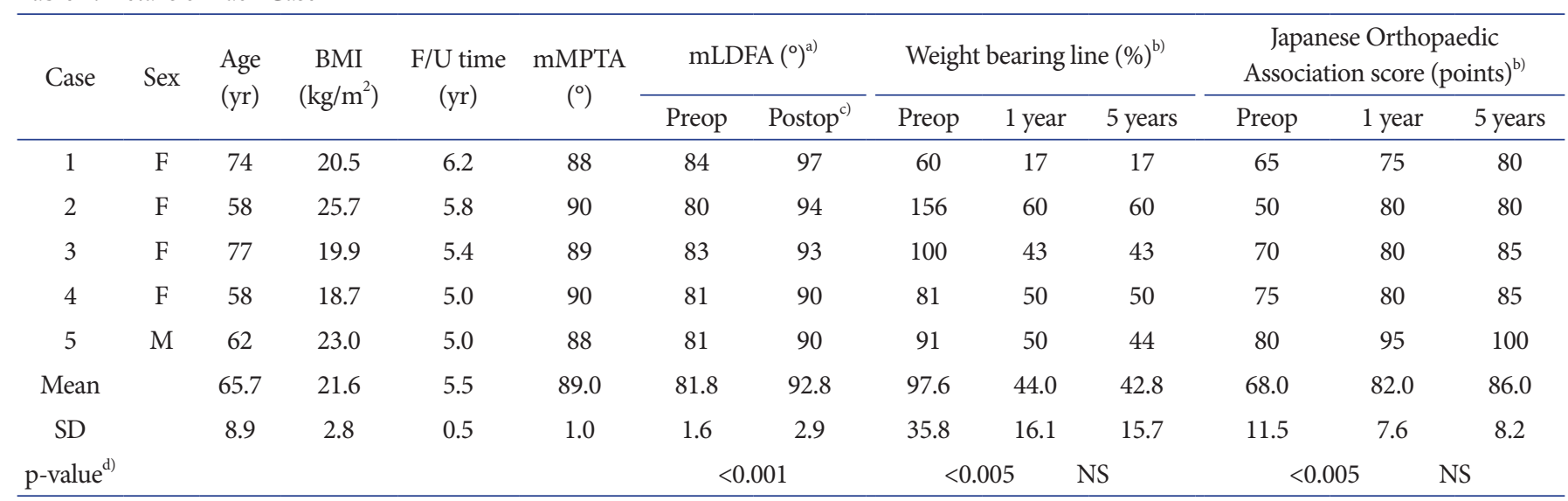

BMI: body mass index, F/U: follow-up, mMPTA: mechanical medial proximal tibial angle, mLDFA: mechanical lateral distal femoral angle, Preop: preoperative, Postop: postoperative, SD: standard deviation, NS: not significant.

a) A paired $t$-test was used to compare preoperative and postoperative values for mLDFA.

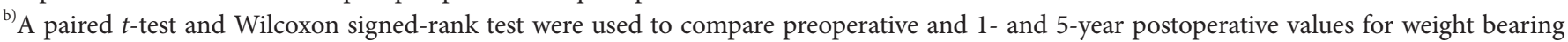
percentage, Japanese Orthopaedic Association score, and range of knee flexion.

${ }^{c}$ Postoperative measurement of the mLDFA was taken 4 weeks after surgery.

${ }^{\mathrm{d})}$ Differences with $\mathrm{p}$-values $<0.05$ were considered statistically significant. 
and mechanical lateral distal femoral angle $(\mathrm{mLDFA})^{6)}$ were $89.0^{\circ} \pm 1.0^{\circ}$ and $81.8^{\circ} \pm 1.6^{\circ}$, respectively (Table 1 ). This means there was no proximal tibial deformity, and all knees had a valgus deformity at the distal femur. The mLDFA was significantly corrected to $92.8^{\circ} \pm 2.9^{\circ}(\mathrm{p}<0.001)$ (Table 1) and it was unchanged for 5 years after CWDFO in all patients (data not shown). This means no bone-related correction loss or overcorrection was noted during the first 5 years postoperatively. The mean WBL percentage and JOA score were corrected from $97.6 \% \pm 35.8 \%$ and $68.0 \pm 11.5$ points to $44.0 \% \pm 16.1 \%$ and $82.0 \pm 7.6$ points ( $\mathrm{p}<0.005$ and $\mathrm{p}<0.05)$, respectively one year after surgery and maintained for the 5-year follow-up period (Table 1). However, the mean range of flexion decreased significantly from $149.0^{\circ} \pm 6.5^{\circ}$ preoperatively to $138.0^{\circ} \pm 5.7^{\circ}$ at 1 year after surgery $(\mathrm{p}<0.001)$. Despite plate removal, the range of flexion improved to the preoperative level in only one case (case 5) at the 5-year follow-up. No patient required conversion to total knee arthroplasty (TKA) during the follow-up period.

In case 1 , there was a $6^{\circ}$ overcorrection compared with preoperative planning. The overcorrection was not noted during surgery despite the intraoperative alignment check using the cable method $^{7}$. At the 5-year follow-up, the patient complained of increased discomfort in the left knee. Arthroscopy was performed and a horizontal tear of the medial meniscus was partially removed. Case 2 demonstrated the most severe valgus deformity, with a WBL percentage of $156 \%$ (Fig. 3A). The mMPTA, joint line convergence angle $e^{6)}$ and mLDFA were $90^{\circ},-10^{\circ}$, and $80^{\circ}$, respectively. Therefore, the deformity was solely caused by the

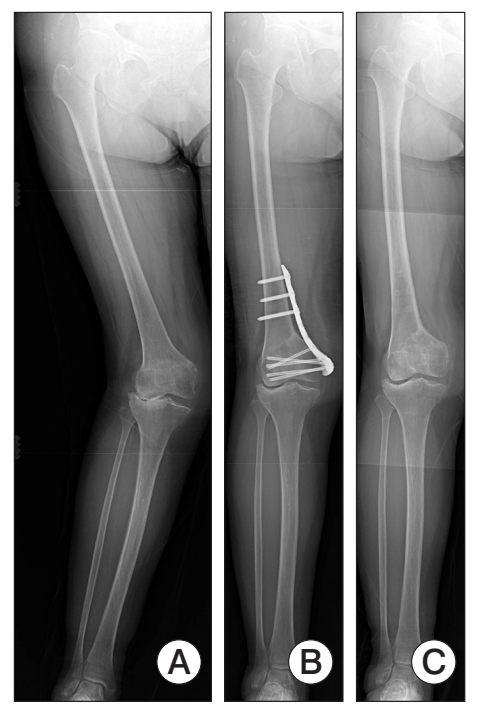

Fig. 3. Case 2. Standing whole-leg radiographs taken preoperatively (A), 1 year postoperatively (B) and 5 years postoperatively $(\mathrm{C})$. distal femur and the remaining problem was severely stretched medial soft tissue laxity. If a TKA were chosen for such cases, a constrained type of TKA would be chosen due to the medial laxity ${ }^{8)}$. Therefore, in spite of the medial and patellofemoral osteoarthritic changes observed on arthroscopy, DFO was selected to avoid a constrained type TKA at a young age. To prevent osteoarthritis progression, we aimed for a corrected WBL percentage of $60 \%$, and the planned alignment was achieved (Fig. 3B). The mLDFA was corrected from $80^{\circ}$ to $94^{\circ}$, and it was preserved during the 5-year follow-up (Fig. 3C). Case 3 suffered an intraoperative lateral cortex fracture. Because bone union was delayed, full weight bearing was postponed until 3 months after surgery. The osteotomized site was completely fused 9 months after surgery, without correction loss or overcorrection. Despite the absence of intraoperative complication, case 4 had a $10^{\circ}$ loss of flexion at the 5-year follow-up as well. The flexion range of cases 1-4 was decreased one year after surgery, resulting in a loss of flexion at the 5-year follow up. In case 5, based on the flexion losses described above, we attempted deep knee flexion in the patient intraoperatively after plate fixation. The medial patellofemoral ligament (MPFL) was too tight during knee flexion because the plate head encroached on the medial femoral epicondyle (Fig. 4). After releasing the MPFL, full knee flexion was achieved and no patellar subluxation was seen intraoperatively.

\section{Discussion}

As the improvement in the JOA score was maintained for 5

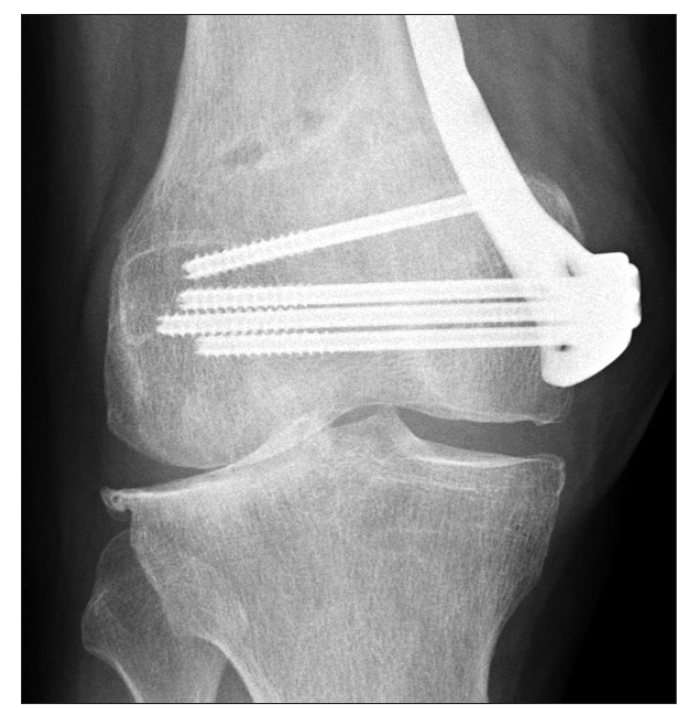

Fig. 4. Case 5. Anteroposterior view showing complete coverage of the medial femoral epicondyle by the plate. 
years without TKA conversion in all cases, CWDFO with the NCB-PT may provide mid-term benefits for patients with lateral OA. Despite good long-term results of TKA for severely valgus knees, achieving adequate soft-tissue balance remains challenging $^{8)}$. There may be limitations to correction of extra-articular bony valgus deformity solely via intra-articular correction during TKA; a constrained prosthesis, which can be used if appropriate balance is not obtained ${ }^{8}$, can result in problems such as implant failure. Therefore, for younger patients like case 2 (Fig. 3), even when there is moderate medial and patellofemoral osteoarthritis, time-saving CWDFO could be a possible option to avoid TKA with a constrained prosthesis.

The healing of the lateral cortex fracture in case 3 required 9 months. Therefore, preserving an intact lateral cortex is crucial to the success of medial CWDFO. For open wedge HTO, a lateral cortex fracture is classified as Takeuchi type I or II". According to the "fibula-as-supporting-strut theory" of Nakamura et al. ${ }^{10)}$, a type I lateral cortex fracture in which the fracture portion is supported by the fibula is considered stable, whereas a type II fracture, in which the fracture portion is not supported by the fibula, is considered unstable ${ }^{10)}$. Because there is no supporting structure similar to the fibula in the distal femur, lateral cortex fractures that occur during CWDFO should always be considered unstable.

More than $10^{\circ}$ of decrease in flexion was found in only one case (case 2) and the other cases showed relatively well-preserved flexion angles. However, the mean flexion range decreased one year after surgery, resulting in permanent loss of flexion in four of our five cases (cases 1-4). Therefore, for more objective evaluation of these results, despite the lack of mid-term follow-up, we investigated six patients treated by CWDFO using a TomoFix MDF with a minimum of 1-year follow-up (range, 1.2 to 2.2 years). In that series, the JOA score improved from $70.0 \pm 13.8$ points to $96.7 \pm 2.6$ points $(\mathrm{p}<0.005$, paired $t$-test $)$ one year after surgery (data not shown in Table). In contrast to CWDFO with the NCB$\mathrm{PT}$, the mean range of flexion also significantly improved from $130.8^{\circ} \pm 3.8^{\circ}$ to $144.2^{\circ} \pm 10.7^{\circ}(\mathrm{p}<0.01$, paired $t$-test $)$ one year after surgery (data not shown in Table). These results might indicate that flexion loss may be a plate-induced complication. Figures 1 and 2 show the difference in plate position between the TomoFix MDF and the NCB-PT. The distal end of the former is located above the medial femoral epicondyle so as not to cover the joint capsule (Fig. 1), while the latter fully covers the medial femoral epicondyle (Fig. 2). This difference may imply that putting a plate on the medial femoral epicondyle causes flexion loss after CWDFO due to the immobilization of the medial capsule and/or
MPFL. The fact that only the patient with MPFL release during CWDFO (case 5) recovered full range of motion may support this hypothesis. Therefore, a capsule-friendly CWDFO plate may be required to acquire a good range of flexion. Even when DFOspecific plates are used, special care should be taken not to place the plate on the medial femoral epicondyle. An intraoperative check of the flexion range may be useful for prevention of the flexion loss.

In conclusion, the use of the NCB-PT in the upside-down position for CWDFO for the treatment of valgus knee deformities provided mid-term benefits for patients with lateral unicompartmental osteoarthritis. Although the number of patients was small for statistical analysis, the outcomes indicate that using the NCBPT plate may cause loss of knee flexion.

\section{Conflict of Interest}

No potential conflict of interest relevant to this article was reported.

\section{References}

1. Stahelin T, Hardegger F, Ward JC. Supracondylar osteotomy of the femur with use of compression. Osteosynthesis with a malleable implant. J Bone Joint Surg Am. 2000;82:712-22.

2. Kosashvili Y, Safir O, Gross A, Morag G, Lakstein D, Backstein D. Distal femoral varus osteotomy for lateral osteoarthritis of the knee: a minimum ten-year follow-up. Int Orthop. 2010;34:249-54.

3. Jockel JA, Erhardt J, Vincenti M, Reissig J, Hoffmann R, Husain B, Tager G, Partenheimer A, Lill H, Gebhard F, Roderer G. Minimally invasive and open surgical treatment of proximal tibia fractures using a polyaxial locking plate system: a prospective multi-centre study. Int Orthop. 2013;37:701-8.

4. Takeuchi R, Ishikawa H, Aratake M, Bito H, Saito I, Kumagai K, Akamatsu Y, Saito T. Medial opening wedge high tibial osteotomy with early full weight bearing. Arthroscopy. 2009;25:46-53.

5. Okuda M, Omokawa S, Okahashi K, Akahane M, Tanaka Y. Validity and reliability of the Japanese Orthopaedic Association score for osteoarthritic knees. J Orthop Sci. 2012;17:750-6.

6. Paley D, Herzenberg JE, Tetsworth K, McKie J, Bhave A. Deformity planning for frontal and sagittal plane corrective osteotomies. Orthop Clin North Am. 1994;25:425-65.

7. Yoon SD, Zhang G, Kim HJ, Lee BJ, Kyung HS. Comparison of cable method and miniaci method using picture archiving 
and communication system in preoperative planning for open wedge high tibial osteotomy. Knee Surg Relat Res. 2016;28:283-8.

8. Ranawat AS, Ranawat CS, Elkus M, Rasquinha VJ, Rossi R, Babhulkar S. Total knee arthroplasty for severe valgus deformity. J Bone Joint Surg Am. 2005;87 Suppl 1:271-84.

9. Takeuchi R, Ishikawa H, Kumagai K, Yamaguchi Y, Chiba N, Akamatsu Y, Saito T. Fractures around the lateral cortical hinge after a medial opening-wedge high tibial osteotomy: a new classification of lateral hinge fracture. Arthroscopy. 2012;28:85-94.

10. Nakamura R, Komatsu N, Murao T, Okamoto Y, Nakamura S, Fujita K, Nishimura H, Katsuki Y. The validity of the classification for lateral hinge fractures in open wedge high tibial osteotomy. Bone Joint J. 2015;97:1226-31. 